\title{
Radiation enhancement and "temperature" in the collapse regime of gravitational scattering
}

\author{
Dimitri Colferai* \\ (Dipartimento di Fisica, Università di Firenze and INFN Sezione di Firenze) \\ E-mail: colferaidfi.infn.it
}

Marcello Ciafaloni

(Dipartimento di Fisica, Università di Firenze)

E-mail: ciafaloniafi.infn.it

\begin{abstract}
We generalize the semiclassical treatment of graviton radiation to gravitational scattering at very large energies $\sqrt{s} \gg m_{P}$ and finite scattering angles $\Theta_{s}$, so as to approach the collapse regime of impact parameters $b \simeq b_{c} \sim R \equiv 2 G \sqrt{s}$. Our basic tool is the extension of the recently proposed, unified form of radiation to the ACV reduced-action model and to its resummed-eikonal exchange. By superimposing that radiation all-over eikonal scattering, we are able to derive the corresponding (unitary) coherent-state operator. The resulting graviton spectrum, tuned on the gravitational radius $R$, fully agrees with previous calculations for small angles $\Theta_{s} \ll 1$ but, for sizeable angles $\Theta_{s}(b) \leq \Theta_{c}=\mathscr{O}(1)$ acquires an exponential cutoff of the large $\omega R$ region, due to energy conservation, so as to emit a finite fraction of the total energy. In the approach-to-collapse regime of $b \rightarrow b_{c}^{+}$we find a radiation enhancement due to large tidal forces, so that the whole energy is radiated off, with a large multiplicity $\langle N\rangle \sim G s \gg 1$ and a well-defined frequency cutoff of order $R^{-1}$. The latter corresponds to the Hawking temperature for a black hole of mass notably smaller than $\sqrt{s}$.
\end{abstract}

The European Physical Society Conference on High Energy Physics

5-12 July

Venice, Italy

* Speaker. 


\section{Outline}

In this talk I show that a quantum description of particle scattering at extreme energies can give rise to graviton radiation whose properties are similar to Hawking radiation, thus suggesting a sort of gravitational collapse. However, the radiation process can still be described by a unitary $S$-matrix, thus pointing towards a resolution of the information paradox.

In order to explain the main ideas of our analysis, I shall give a brief introduction to the ACV method for describing string or particle collisions at transplanckian energies; then I'll describe our method for treating graviton radiation which is based on a unified emission amplitude. Finally I'll show and discuss the main results: (i) the final state radiation produced in such collisions is a unitary pure state; (ii) we see the role of the gravitational radius in the graviton spectrum, since $\langle\omega\rangle \sim 1 / R$; (iii) the ensuing energy spectrum presents a "quasi-temperature" $T$ of the order of the Hawking temperature $T_{H}$.

\section{The ACV approach}

Following the pioneering work of Amati-Ciafaloni-Veneziano (ACV) [四, we consider two strings colliding at center-of-mass energy $2 E=\sqrt{s} \gg M_{P}$ much larger than the Planck mass, with some impact parameter $b$. The condition on the energy could lead, according to general relativity, to the formation of a macroscopic black-hole whose radius $R=2 G \sqrt{s} \gg l_{P}, l_{s}$ is much larger than both the Planck length and the string length. In this high-energy regime the adimensional parameter $\alpha_{G} \equiv G s / \hbar$ is very large and plays the role of effective gravitational coupling. It measures also the typical action of such processes, in units of $\hbar$.

ACV found that string scattering amplitudes at transplanckian energies and at fixed momentum transfer (the so called Regge limit) can be interpreted as effective ladder-like diagrams involving (Reggeized) graviton exchanges between (excited) string states.

Since strings are extended objects, they interact by exchanging soft momenta, of the order $\hbar / b$. On the other hand, they can exchange a large number of quanta, on average $\langle n\rangle=G s / \hbar$. In this way the overall exchanged momentum can be large: $Q \sim G s / b$ yielding a finite deflection angle $\Theta_{E}=Q / E \sim R / b$.

In the semiclassical regime $b \gtrsim R \gg l_{P}$, the string states are actually not excited, i.e., onshell, and can be treated as point particles. The generic ladder diagram is then just a convolution in transverse momentum of the single rung amplitude. This convolution can be diagonalized by Fourier transform from transverse momentum $Q$ to impact parameter space $b$. The resummation of such diagrams yields an exponential series, and provides the "eikonal" form of the $S$-matrix for elastic scattering $S_{\mathrm{el}}=\mathrm{e}^{\mathrm{i} 2 \delta(b, s)}$, where $\delta(b, s) \equiv \alpha_{G} \Delta(b)$ represents the phase shift. From the latter, one can compute some observables, e.g., the Einstein deflection angle $\Theta_{E}=\sqrt{s} /(4 \hbar) \partial \delta / \partial b=$ $2 R / b$.

In order to approach the collapse region, we decrease the impact parameter at values $b \sim R$. Here gravity is strong and subleading corrections to the eikonal (of order $R^{2} / b^{2}$ ) become important. ACV were able to identify such subleading diagrams [ [ $]$, the so-called $\mathrm{H}$ diagrams.

The new ingredient of such diagrams is an effective vertex introduced by Lipatov [ [ $]$ ]. By resumming also the subleading diagrams, ACV determined the next-to-leading corrections to the 
phase shift and found a very interesting feature [四]: for impact parameters smaller than a critical value $b_{c} \sim R$, the phase shift acquires a positive imaginary part, implying a suppression of the elastic $S$-matrix. In particular, at vanishing impact parameters, the elastic $S$-matrix is exponentially suppressed like $\left|S_{\mathrm{el}}\right| \sim \exp (-\pi G s / \hbar)$.

A crucial question then arises: is such elastic suppression fully compensated by inelastic graviton production which restores unitarity? Or is such critical parameter the signal of something deeper, maybe the signal of a gravitational collapse? Note that $b_{c}$ is a branch-cut singularity of $\Delta(b)$, with a fractional critical exponent $3 / 2$.

\section{Graviton radiation in transplanckian scattering}

The Lipatov vertex is appropriate only in the so-called multi-Regge kinematics, where the emission angle of the graviton is much greater than the scattering angle of the external particle. In the complementary region of small emission angles and relatively soft graviton energies $(\omega \ll E)$, amplitudes are well described by the Weinberg current. Those two regions overlap, and we can write a unified amplitude [ [D] describing in a simple and elegant way graviton emission in both Regge region and soft region. In particular, in coordinate space, where $b$ is the impact parameter and $x$ the transverse coordinate of the emitted graviton, such amplitude can be expressed as the soft amplitude, minus the same soft amplitude with $E$ (the energy of the external particle) replaced by $\omega$ (the energy of the emitted graviton):

$$
\mathscr{M}_{\lambda}=\sqrt{\alpha_{G}} \frac{R}{2 \pi^{2}} \mathrm{e}^{\mathrm{i} \lambda \phi_{q}} \int \frac{\mathrm{d}^{2} x}{|x|^{2} \mathrm{e}^{\mathrm{i} \lambda \phi_{x}}} \mathrm{e}^{\mathrm{i} q \cdot x}\left\{\frac{E}{\omega}\left[\Delta(b)-\Delta\left(b-\frac{\omega}{E}\right)\right]-[\Delta(b)-\Delta(b-x)]\right\} .
$$

We can interpret these two contributions as graviton insertions on the external legs (à la Weinberg) and on the internal leg, respectively. We refer to such decomposition as "soft-based" representation.

The single-graviton emission amplitude is then computed by resumming all ladder diagrams with one emission. When gravitons are emitted from within the ladder, the local incoming particle momentum is rotated with respect to the $z$-axis. Accordingly, amplitudes acquires phase factors. Denoting with $\theta\left(\theta_{s}\right)$ the graviton emission angle (the scattering angle), if $\theta_{s} \ll \theta$, such phase factors cancel and amplitudes interfere constructively. On the other hand, if $\theta \sim \theta_{s}$, this is no more the case, and coherence is lost. Quantitatively this has very important consequences.

In addition, the emitted graviton can rescatter with the incident particles. In particular, a graviton at transverse position $x$ mainly interacts with the particle moving in the opposite direction at transverse position $b$, and the interaction is given by the usual eikonal function, but now proportional to the graviton energy omega and with shifted transverse distance $b-x$.

It turns out that the full $2 \rightarrow 3$ amplitude can be written as the elastic amplitude, times a factor $\mathfrak{M}$ which describes graviton production:

$$
\mathfrak{M}_{\lambda}(b ; \omega, q)=\sqrt{\alpha_{G}} \frac{R \mathrm{e}^{\mathrm{i} \lambda \phi_{q}}}{2 \pi^{2}} \int \frac{\mathrm{d}^{2} x}{|x|^{2} \mathrm{e}^{\mathrm{i} \lambda \phi_{x}}} \frac{\mathrm{e}^{\mathrm{i} q \cdot x}}{2 \mathrm{i} \omega R}\left\{\mathrm{e}^{2 \mathrm{i} \omega R[\Delta(b-x)-\Delta(b)]}-\mathrm{e}^{2 \mathrm{i} \omega R \frac{E}{\omega}\left[\Delta\left(b-\frac{\omega}{E} x\right)-\Delta(b)\right]}\right\}
$$

The first term contains the effect of angular coherence discussed before, the second term contains the rescattering effects. It is important to note that the same $\Delta$ function describes eikonal exchanges during the elastic scattering and graviton production and rescattering! 
The final step is to consider the emission of an arbitrary number of gravitons. In the eikonal approximation such emissions are independent. In fact, for $b \gg R$, we have shown that the amplitude for the emission of $N$ real gravitons factorizes as a product of the elastic amplitude times an emission factor $\mathfrak{M}_{\lambda_{i}}\left(q_{i}\right): i=1, \cdots, N$ for each graviton. Such factorization is equivalent to assume that the $S$-matrix is given by the elastic $S$-matrix times a coherent state operator acting on the Fock space of gravitons: $S=\mathrm{e}^{\mathrm{i} 2 \delta} \exp \left\{\mathrm{i} \sum_{\lambda} \int \frac{\mathrm{d}^{3} k}{2 \omega_{k}} \mathfrak{M}_{\lambda}(k) a_{\lambda}^{\dagger}(k)+\right.$ h.c. $\}$. The $a^{\dagger}$ term in the exponent is responsible for graviton creation via the emission factor $\mathfrak{M}$, and we add the hermitian conjugate term which effectively describes virtual corrections, according to Weinberg analysis of soft graviton emission. In this way we have a unitary $S$-matrix describing transplanckian collisions.

Using the previous result it is straightforward to compute the energy or frequency $(\hbar=1)$ spectrum of graviton radiation [[]]. It is shown in fig. Пa, for various values of the scattering angle, i.e., of the ratio $R / b$. The overall radiated energy increases with $s$ and also with the scattering angle. However, the shape of the spectrum is weakly dependent of such kinematical parameters: it is almost constant for small frequencies $\omega<1 / R$, reproducing the expected zero-frequency limit, while it decreases like $1 / \omega$ for $\omega>1 / R$. The main feature of this spectrum is that is clearly shows a characteristic frequency $\omega \sim 1 / R$, almost independently of the scattering angle. This means that if we increase the energy of the process, the frequency of the radiation decreases, in a way reminiscent of how Hawking radiation depends upon the black-hole mass.
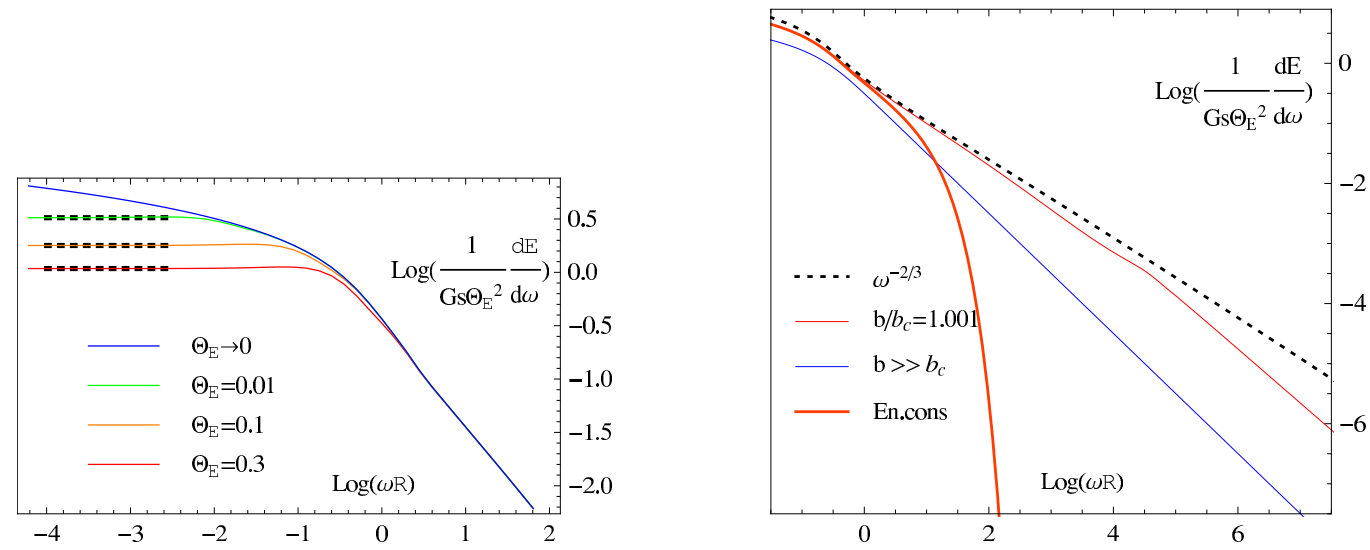

Figure 1: Left (a): graviton energy spectrum for $b \gg R$, i.e., small deflection angles. Right (b): spectrum close to the critical parameter $b \simeq b_{c}$ with and without energy conservation.

Now we want to move towards the strong coupling region $b \sim R$. Here, we have to take into account (i) $\mathrm{H}$ diagrams along the ladder and (ii) graviton emissions from $\mathrm{H}$ diagrams. The first effect is easily taken into account, by replacing the leading eikonal phase $\Delta$ with the resummed one that develops an imaginary part at $b=b_{c}$. The second effect is more difficult to evaluate. We argue [6] that the sum of external and internal insertions of gravitons into the $\mathrm{H}$ diagram can be again described by the "soft-based" representation mentioned before.

By neglecting correlations between emitted gravitons, we end up with the same formulae for amplitudes and spectra, where now the $\Delta$ function is the resummed one. For $b$ approaching $b_{c}$, we note a significant enhancement of radiation. In particular, there is a region where the spectrum decreases slowly, like $\omega^{-2 / 3}$, before eventually decreasing like $1 / \omega$ (fig. (Wb). This is due to larger and larger tidal forces as $b$ decreases, and an increasing fraction of energy is radiated off. 


\section{Energy conservation and "temperature"}

At this point it is important to take into account at least those correlations due to energy conservation. We impose it event by event, by requiring $\sum_{i} \omega_{i}<E$, and extend this bound to virtual corrections on the basis of AGK cutting rules. Without entering into technical details, we insert the step function $\Theta\left(E-\sum_{i} \omega_{i}\right)$ in the individual emission probabilities. By using the usual integral representation for the step function, we find that the integral is dominated by a saddle point $\bar{\tau}$, and the result [司] is that the naive uncorrelated spectrum is suppressed by a factor $\mathrm{e}^{\omega / \bar{\tau}}$ (fig. $\mathrm{Db}$ ). This behaviour is similar to thermal radiation, and in our case the quasi-temperature approaches $T=1 /(\bar{\tau} R)=0.8 / R \sim T_{H}$ at $b=b_{c}$, say the temperature of a black-hole of mass $\simeq 0.1 \sqrt{s}$. But I want to stress that our radiation is coherent, it is a pure state!

Finally we want to cross the critical impact parameter and reach $b \ll R$, i.e., central collisions. In this case the elastic amplitude provides an exponential suppression $\exp (-\pi E R)$ of all graviton amplitudes. However, the rescattering term of the emission factor, which dominates in this regime, is enhanced like $\exp (+\pi \omega R)$. Therefore, if $\sum_{j} \omega_{j}=E$, such enhancement compensates the suppression, and there is the possibility of recovering unitarity also in this subcritical regime. Here analytic and numerical calculations are more difficult, but a first estimate of the quasi-temperature indicates a value $T \simeq 0.7 / R$, similar to that found at $b=b_{c}$ and again of the order of $T_{H}$.

In conclusion, we set up a framework which allows us to compute graviton radiation in transplanckian collisions. We now see the role of the gravitational radius as the inverse of the characteristic frequency for all values of center-of-mass energies and impact parameters. When the impact parameter is $b \lesssim R$, tidal forces cause a dramatic enhancement of graviton radiation and all energy is radiated off. By taking into account energy-conservation constraints, we obtain a coherent radiation pattern with an exponentially decreasing spectrum, resembling a thermal radiation with $T \sim T_{H}$ of a black-hole somewhat lighter than $\sqrt{s}$. All in all, this radiation mechanism preserve unitarity and suggests a possible solution of the information paradox.

\section{References}

[1] D. Amati, M. Ciafaloni and G. Veneziano, Superstring Collisions at Planckian Energies, Phys. Lett. B 197 (1987) 81.

[2] D. Amati, M. Ciafaloni and G. Veneziano, Higher Order Gravitational Deflection and Soft Bremsstrahlung in Planckian Energy Superstring Collisions, Nucl. Phys. B 347 (1990) 550.

[3] L. N. Lipatov, High-energy scattering in QCD and in quantum gravity and two-dimensional field theories, Nucl. Phys. B 365 (1991) 614.

[4] D. Amati, M. Ciafaloni and G. Veneziano, Towards an S-matrix description of gravitational collapse JHEP 0802 (2008) 049 [hep-th/ 0712 . 1209].

[5] M. Ciafaloni, D. Colferai and G. Veneziano, Emerging Hawking-Like Radiation from Gravitational Bremsstrahlung Beyond the Planck Scale, Phys. Rev. Lett. 115 (2015) no.17, 171301

[hep-th/1505.06619].

M. Ciafaloni, D. Colferai, F. Coradeschi and G. Veneziano, Unified limiting form of graviton radiation at extreme energies, Phys. Rev. D 93 (2016) no.4, 044052 [hep-th/1512 . 00281].

[6] M. Ciafaloni and D. Colferai, Radiation enhancement and temperature in the collapse regime of gravitational scattering, Phys. Rev. D 95 (2017) no.8, 086003 [hep-th/1612 . 06923 ]. 